\title{
Artificial intelligence system can achieve comparable results to experts for bone age assessment of Chinese children with abnormal growth and development
}

\author{
Fengdan Wang ${ }^{\text {Equal first author, } 1}$, Xiao Gu ${ }^{\text {Equal first author, } 2}$, Shi Chen ${ }^{2}$, Yongliang Liu ${ }^{3}$, Qing Shen ${ }^{3}$, Hui Pan ${ }^{\text {Corresp., } 2}$, Lei Shi \\ Corresp., 3 , Zhengyu Jin ${ }^{1}$ \\ ${ }^{1}$ Department of Radiology, Peking Union Medical College Hospital, Chinese Academy of Medical Sciences \& Peking Union Medical College, Beijing, China \\ 2 Department of Endocrinology, Peking Union Medical College Hospital, Chinese Academy of Medical Sciences \& Peking Union Medical College, Beijing, \\ China \\ 3 Hangzhou YITU Healthcare Technology Co., Ltd., Hangzhou, China \\ Corresponding Authors: Hui Pan, Lei Shi \\ Email address: wangfengdan@pumch.cn, lei.shi@yitu-inc.com
}

Objective. Bone age (BA) is a crucial indicator for revealing the growth and development of children. This study tested the performance of a fully automated artificial intelligence (AI) system for BA assessment of Chinese children with abnormal growth and development.

Materials and methods. A fully automated Al system based on the Greulich and Pyle (GP) method was developed for Chinese children by using 8,000 BA radiographs from five medical centers nationwide in China. Then, a total of 745 cases ( 360 boys and 385 girls) with abnormal growth and development from another tertiary medical center of north China were consecutively collected between January and October 2018 to test the system. The reference standard was defined as the result interpreted by two experienced reviewers (a radiologist with 10 years and an endocrinologist with 15 years of experience in BA reading) through consensus using the GP atlas. BA accuracy within 1 year, root mean square error (RMSE), mean absolute difference (MAD), and 95\% limits of agreement according to the Bland-Altman plot were statistically calculated.

Results. For Chinese pediatric patients with abnormal growth and development, the accuracy of this new automated Al system within 1 year was $84.60 \%$ as compared to the reference standard, with the highest percentage of $89.45 \%$ in the 12 - to 18 -year group. The RMSE, MAD, and $95 \%$ limits of agreement of the Al system were 0.76 years, 0.58 years, and -1.547 to 1.428 , respectively, according to the BlandAltman plot. The largest difference between the Al and experts' BA result was noted for patients of short stature with bone deformities, severe osteomalacia, or different rates of maturation of the carpals and phalanges.

Conclusions. The developed automated Al system could achieve comparable BA results to experienced reviewers for Chinese children with abnormal growth and development. 


\section{Artificial intelligence system can achieve comparable results to experts}

2 for bone age assessment of Chinese children with abnormal growth and

3 development

5 Fengdan Wang ${ }^{1 \mathrm{a}}, \mathrm{Xiao} \mathrm{Gu}^{2 \mathrm{a}}$, Shi Chen ${ }^{2}$, Yongliang Liu ${ }^{3}$, Qing Shen ${ }^{3}$, Hui Pan ${ }^{2 *}$, Lei Shi ${ }^{*}$, Zhengyu

$6 \operatorname{Jin}^{1}$

7

8

${ }^{1}$ Department of Radiology, Peking Union Medical College Hospital, Chinese Academy of Medical

9 Sciences \& Peking Union Medical College, Beijing, China

2 Department of Endocrinology, Peking Union Medical College Hospital, Chinese Academy of

Medical Sciences \& Peking Union Medical College, Beijing, China

${ }^{3}$ Hangzhou YITU Healthcare Technology Co., Ltd., Hangzhou, China

* Corresponding authors:

Hui Pan, Department of Endocrinology, Peking Union Medical College Hospital, No.1 Shuaifuyuan, Dongcheng District, Beijing, China 100730. Phone: +86-10-6915 5842. Email: wangfengdan@pumch.cn.

Lei Shi, Hangzhou YITU Healthcare Technology Co., Ltd., Xihu District, Hangzhou, China 310000.

Phone: +86-0-133 8581 7768. Email: lei.shi@yitu-inc.com.

21

a The authors contributed equally to this paper and were recommended as first authors. 


\section{Artificial intelligence system can achieve comparable results to} experts for bone age assessment of Chinese children with abnormal growth and development

\section{ABSTRACT}

Objective. Bone age (BA) is a crucial indicator for revealing the growth and development of children. This study tested the performance of a fully automated artificial intelligence (AI) system for BA assessment of Chinese children with abnormal growth and development. Materials and methods. A fully automated Al system based on the Greulich and Pyle (GP) method was developed for Chinese children by using 8,000 BA radiographs from five medical centers nationwide in China. Then, a total of 745 cases ( 360 boys and 385 girls) with abnormal growth and development from another tertiary medical center of north China were consecutively collected between January and October 2018 to test the system. The reference standard was defined as the result interpreted by two experienced reviewers (a radiologist with 10 years and an endocrinologist with 15 years of experience in BA reading) through consensus using the GP atlas. BA accuracy within 1 year, root mean square error (RMSE), mean absolute difference (MAD), and 95\% limits of agreement according to the Bland-Altman plot were statistically calculated.

Results. For Chinese pediatric patients with abnormal growth and development, the accuracy of this new automated $\mathrm{Al}$ system within 1 year was $84.60 \%$ as compared to the reference standard, with the highest percentage of $89.45 \%$ in the 12 - to 18 -year group. The RMSE, MAD, and $95 \%$ limits of agreement of the Al system were 0.76 years, 0.58 years, and -1.547 to 1.428 , respectively, according to the Bland-Altman plot. The largest difference between the Al and experts' BA result was noted for patients of short stature with bone deformities, severe osteomalacia, or different rates of maturation of the carpals and phalanges.

Conclusions. The developed automated Al system could achieve comparable BA results to experienced reviewers for Chinese children with abnormal growth and development. 
52

53

54

55

56

\section{INTRODUCTION}

Bone age (BA), which evaluates skeletal maturity from the radiographs of the left hand and wrist, is a crucial indicator for revealing the growth and development of children (Creo \& Schwenk, 2017). Two methods are mainly used to assess BA: the Greulich and Pyle (GP) and Tanner-Whitehouse (TW3) (Greulich \& Pyle, 1959; Tanner, 1962; Tanner et al., 1983; Tanner et al., 2001); of these, the GP atlas is generally accepted as a faster and simpler method and thus widely applied in clinical practice (De Sanctis et al., 2014). However, manual assessment of BA completely depends on the reviewers' experience to determine BA (Creo \& Schwenk, 2017; Mari, 2015), thereby causing significant intra- and inter-observer variations. Furthermore, constant time and effort are needed to train clinical reviewers; consequently, primary and rural hospitals face a daunting task to carry out this important examination.

Artificial intelligence (Al), which has high potential in reducing labor requirement and intra- and inter-observer variations, is gaining popularity in medical field, especially in radiology (Cheng et al., 2019; Hosny et al., 2018). Deep learning, one of the advanced Al techniques, which can automatically learn features from images, has become a hot spot in recent years. BA images are an ideal database to train deep learning algorithms, because bone radiographs contain black-white-gray gradations that show variations (Hu et al., 2017).

In addition to some traditional learning-based approaches (van Rijn \& Thodberg, 2013; Thodberg et al., 2017), several preliminary deep learning-based BA systems have been developed using a standard database or radiographs from one or two medical centers in North America and Korea (Kim et al., 2017; Spampinato et al., 2017; Larson et al., 2018; Tajmir et al., 2019). However, these deep learning-based systems were developed for Western and Korean populations; thus, they might not be suitable for Chinese children. Moreover, the number of tested patients was relatively small and only with limited types of diseases. Therefore, these Al BA systems cannot be directly applied to populations of different ethnicities, and a fully automated Al system for BA assessment of Chinese children should hence be developed. In addition, the test sample should be larger and should include patients with a wide range of diseases, because children with abnormal growth and development are more likely to have impaired bone maturation and malformations that pose greater challenge for Al. 
81

82

Herein, a fully automated AI BA system for Chinese children based on the GP method was developed using 8000 BA radiographs from five medical centers nationwide in China (Zhou et al., 2019, unpublished data). This study aimed to evaluate the performance of this Al system for BA assessment of Chinese children with endocrine disorders. The test cases used to validate this Al system were patients with abnormal growth and development and were consecutively recruited from another tertiary medical center in north China.

\section{SUBJECTS AND METHODS}

\section{Model implementation}

This study was approved by the Institutional Review Board of Peking Union Medical College Hospital (Approval No. S-K978), and informed consent was waived because this was a retrospective study. The patients were anonymized by de-identifying their personal information prior to analysis. Between January 2012 and December 2016, 8000 left-hand radiographs from five different medical centers nationwide in China were used as input data for developing the Al model (Zhou et al., 2019, unpublished data). Our medical center is not one of the five medical centers that provided the radiographs. The demographic characteristics of the 8000 cases (training dataset of 7000 cases, validation dataset of 760 cases, and test dataset of 240 cases) are shown in Supplementary Materials (Fig. S1 \& Table S1). The BA results extracted from the original radiology reports were used as the reference standard.

The Al model consisted of an alignment module and a subsequent classification module (Fig. 1). The two modules were built on the same architecture, known as a deep residual network (ResNet), which is a deep convolutional neural network (CNN) with 50 layers and approximately $3.6 \times 10^{9}$ floating point operations (FLOPS). The model was implemented using an open-source machine learning library (TensorFlow version 1.4.1; Google, Mountain View, CA, USA). The model was trained on an Ubuntu 16.04 computer with 14 Intel Xeon CPUs, using a NVIDIA GTX 1080 Ti 11 Gb GPU, with 256 GB random access memory (RAM).

Before training, each radiograph was first converted from DICOM to portable network graphic (PNG) file format. The radiographs contained images of distal ulna, distal radius, left wrist, and metacarpal and phalangeal bones with a resolution of at least $1000 \times 1000$ pixels 
110 formatted using Python (version 3.7) and the pydicom library (Python Software Foundation;

111

112

113

114

115

116

117

118

119

120

121

122

123

124

125

126

127

128

129

130

131

132

133

134

135

136

137

138

version 0.9.9, Beaverton, OR, USA). The images were further downsized to $256 \times 256$ pixels by using Python image library.

After pre-processing of the clinical radiographs, the alignment module adopts a ResNet to directly regress all the coordinates of 59 localized points. These characteristics are used for determining the bounding area of the concerned bones and ossification center. The region of interest (ROI) can then be extracted from hand radiographs, and the classification module can extract features from the concerned bones and ignore the region unnecessary for BA assessment.

Training of the model was performed by stochastic gradient descent in batches of 20 images per step by using an Adam Optimizer with a learning rate of 0.001 . Training on all categories was run for 80,000 iterations, because training of the final layers will have converged by then for all classes. After 80,000 iterations through the entire dataset, the training was stopped due to the absence of further improvement in both accuracy and sigmoid loss.

\section{Test patient population}

From January to October 2018, left-hand BA radiographs were consecutively collected from 753 pediatric patients aged 4 to 18 years who presented to our medical center with a complaint of abnormal growth and development. Except for this criterion, we chose patients without any age and sex preference. Among the 753 patients, radiographs of eight patients were excluded because of poor imaging quality. Finally, a total of 745 radiographs (male to female ratio $=$ 1:1.07) were included to test the Al system. The flowchart of case collection is shown in Fig. 2. The de-identified DICOM images were downloaded from the PACS system and then inputted into the Al system for batch processing. The Al system automatically generated the BA results.

\section{Reference standard}

The reference standard was provided by two trained and experienced reviewers (a radiologist with 10 years and an endocrinologist with 15 years of experience in BA reading) using the GP 
139

140

141

142

143

144

145

146

147

148

149

150

151

152

153

154

155

156

157

158

159

160

161

162

163

164

165

166

167

atlas. Both reviewers assessed all the 745 cases through consensus. They were blinded to patient information, diagnosis, treatment, and previous BA reports apart from sex and age.

There was no time limit to assess all radiographs. In the case of a disagreement, a third reviewer, an endocrinologist specialized in child growth and development with over 20 years of experiences in BA reading, was consulted.

Regarding the GP atlas, "Skeletal development of the hand and wrist-a radiographic atlas and digital BA companion" published by Oxford University Press in 2011 was adopted. This digital atlas has been widely used in clinical practice as an efficient and accurate method (Bunch et al., 2017).

\section{Statistical analysis}

To compare the evaluation results of the Al system and human reviewers, several statistical variants were used. BA accuracy was defined as the percentage of the differences between the two methods within 1 year. Pearson's correlation coefficient was calculated to analyze the relativity. Bland-Altman plots were used to calculate the mean and $95 \%$ confidence interval of the difference between them. Root mean square error (RMSE) and median absolute deviation (MAD) were quantified to evaluate the accuracy. Statistical differences were considered to be significant at $p<0.05$. Calculations were performed using SPSS v.22.0 software (SPSS Inc., Chicago, IL, USA), and Bland-Altman plots were created using GraphPad Prism 7 statistical software (Graphpad Software, San Diego, CA, USA).

\section{RESULTS}

\section{Test patient population}

Between January and October 2018, a total of 745 BA radiographs were included to test the Al BA software. All radiographs were obtained from Chinese children with abnormal growth and development. The demographic information of the 745 patients is presented in Table 1. There were 360 males and 385 females, with a mean age of 10.2 years (range: 4-18 years). Most patients $(420 / 745,56.38 \%)$ were included in the 6- to 12-year group, followed by 199 patients $(26.71 \%)$ in the 12 - to 18 -year group and 126 patients (16.91\%) in the $4-6$-year-old group. The 
168

169

170

171

172

173

174

175

176

177

178

179

180

181

182

183

184

185

186

187

188

189

190

191

192

193

194

195

196

main reasons for presentation to the clinic were short stature, precocious puberty, macrosomia, and congenital disorders.

\section{Accuracy of the automated AI BA system for Chinese children}

For reading one radiograph, the two experienced reviewers took approximately 2 min on average, while the Al model required only 1 to $2 \mathrm{~s}$. Consequently, the two reviewers spent over $24 \mathrm{~h}$ analyzing the 745 cases, while the Al system batch processed all the images together generating the output in an excel in less than $1 \mathrm{~h}$; this showed that the Al system was significant efficient compared to manual analysis.

By using the two experienced reviewers' manual reading results as a reference standard, the overall BA accuracy of Al within 1 year was $84.60 \%$. The distribution of BA accuracy of Al within 1 year is illustrated in Fig. 3, and one example of a case is shown in Fig. 4A. If we categorized the results into the three age groups $(4-6,6-12$, and $12-18$ years), the highest percentage of BA accuracy within 1 year was $89.45 \%$ in the 12 - to 18 -year.

In addition, the agreement of the AI BA results with the reference standard was further quantified using RMSE, MAD, and the Bland-Altman plot. The RMSE of Al was 0.76 years and the MAD was 0.58 years ( $95 \%$ confidence interval, 0.55 to 0.62 years) when compared with the manual reference standard. The $95 \%$ limits of agreement of the Al system and the reference standard was -1.547 to 1.428 according to the Bland-Altman plot (Fig. 5).

\section{Analysis of the largest deviation}

Five cases with a deviation of $\geq 2$ years were noted between the $\mathrm{Al}$ and manual BA results. To further understand the confounders that caused this deviation, their clinical features and BA radiographs were further analyzed; the results are shown in Table 2 and Fig. 4 B to D.

For patient No. 1, the deformity of the left fifth middle phalange was identified. For patient No. 2, the first inter-phalangeal joint curved and thus obscured the fusion line of the distal phalangeal joint; the epiphysis of the first distal phalangeal joint was the only one with fusion in this boy, which served as an important benchmark to determine the BA according to the GP atlas. In these two cases, the reviewers could identify the deformity and malposition 
197

198

199

200

201

202

203

204

205

206

207

208

209

210

211

212

213

214

215

216

217

218

219

220

221

222

223

224

225

easily, while the Al system could not.

Patient No. 3 received long-term treatment with corticosteroids for systemic juvenile idiopathic arthritis, which led to severe osteomalacia of hand and wrist bones and irregular shapes of the carpal bones. For patients Nos. 4 and 5, the rates of maturation of the carpals and phalanges were different. In accordance with the tips of the GP atlas, the phalanges were given more priority than carpal bones by the reviewers. Therefore, achieving precise BA results for these three cases was difficult for both the experienced reviewers and the Al system.

If these five cases were excluded, the overall BA accuracy of the Al system within 1 year was elevated to $85.10 \%$, the RMSE decreased to 0.73 years, and the MAD was 0.57 years ( $95 \%$ confidence interval, 0.54 to 0.61 years). Additionally, the $95 \%$ limits of agreement of the $\mathrm{Al}$ system and the reference standard was -1.494 to 1.379 according to the Bland-Altman plot.

\section{DISCUSSION}

In this study, we trained a fully automated Al system to assess BA of Chinese children by using 8,000 radiographs from five different medical centers nationwide in China. We then evaluated the performance of the Al system by using 745 radiographs of patients with abnormal growth and development from another medical center. Compared to the interpretation results of experienced human reviewers, the overall BA accuracy of Al within 1 year was nearly $85 \%$. According to the results of RMSE, MAD, and 95\% limits of agreement, the degree of dispersion was also acceptable, indicating that this Al system performs well as a reliable and convenient tool for BA assessment of Chinese children in terms of saving time and energy.

Al systems based on deep learning have a bright future for BA assessment clinically. Some computer-aided programs of BA estimation have been proposed, including analyzing bones using the GP method (Hsieh et al., 2007) and establishing an algorithm based on the TW3 method (Liu et al., 2008; van Rijn \& Thodberg, 2013). However, only in recent years, with the development of deep learning, the research on BA assessment has entered a new era, and several Al systems have been developed to assess BA in North America and Korea. BoneXpert, a traditional machine learning commercial system, is based on a feature extraction technique that reconstructs the borders of the bones. It is reported that the MAD between this system 
226

227

228

229

230

231

232

233

234

235

236

237

238

239

240

241

242

243

244

245

246

247

248

249

250

251

252

253

254

and manual assessments ranged from 0.55 to 0.76 years (van Rijn \& Thodberg, 2013).

Nevertheless, not all the bones in one BA radiograph are considered, and the system cannot identify pathological conditions such as malformations (Kim et al., 2017). In 2017, Kim et al. developed an AI BA system that provided three most likely estimated BA results for one radiograph based on cases from the Asan Medical Center and then tested it on 200 cases evenly distributed by age from the same medical center; the authors obtained a first rank accuracy of 69.5\% (Kim et al., 2017). In 2018, 200 sex-stratified cases from Stanford University, one of the two American medical centers where Larson et al. trained their Al BA system, were used to test its accuracy; the system achieved an RMSE of 0.63 years (Larson et al., 2018). In 2019, Tajmir et al. tested their AI BA system using 280 cases comprising 10 representative cases for each class and sex and chosen from 8,325 radiographs which they used to train the system; the BA accuracy was 73.2\% (Tajmir et al., 2019). Herein, we tested our fully automated AI BA model with radiographs from 745 Chinese pediatric patients with abnormal growth and development. This number of test patients (745 cases) was almost triple of that used in previous studies (Kim et al., 2017; Larson et al., 2018; Booz et al., 2019; Tajmir et al., 2019) and also the largest one for Chinese population. The results showed that this AI BA system for Chinese children exhibited similar accuracy to the experienced reviewers.

Prior studies of BA assessment based on deep learning usually trained and tested the program with radiographs from the same database with similar epidemiological characteristics (Kim et al., 2017; Larson et al., 2018; Tajmir et al., 2019). However, real clinical circumstances are more complicated, as the Al program is supposed to be applied in various medical centers facing patients with a wide range of diseases. Our training data set was chosen from five different medical centers in five different cities nationwide in China, and the test cases were from a sixth medical center in north China. The use of radiographs from different places for algorithm development and program evaluation could simulate the clinical application of the Al program. Moreover, our cohort was consecutively selected without age or sex preference, which was more like a mimic of clinical situation.

Of note, the performance of our algorithm was best for children aged between 12 to 18 years. Skeleton development is more mature for the older children in the 12-18 years age group 
255 because all the 29 bones of the hand have developed. For younger children, the appearance of

256

257

258

259

260

261

262

263

264

265

266

267

268

269

270

271

272

273

274

275

276

277

278

279

280

281

282

283

hand bones varies; thus, there were less ROls as input for extracting features and BA

assessment in the radiographs of younger children. Consequently, the accuracy of AI BA assessment for children younger than 12 years may be less accurate than that for children aged 12-18 years. In clinical practice, BA assessment for children younger than 12 years is also more difficult for radiologists and endocrinologists, and interobserver variability is more likely to occur (Ebrahimzade et al., 2019; Alshamrani, Messina \& Offiah, 2019). Therefore, more training data of left hand radiographs from younger children are helpful to improve the performance of the AI BA system.

The phalanges and metacarpal, and carpal bones grew and developed differently in two cases of the 5 patients with a deviation of over 2 years between the Al system and the reference standard; this made the hand radiograph resemble several standard GP images obtained at different ages, thus causing the difficulty in BA assessment for both AI and experienced human readers (Molinari, Gasser \& Largo, 2004; Zhang et al., 2008). This unsynchronized bone growth may result from certain diseases or abnormal secretion of hormones (Kim et al., 2010; Polito et al., 1994). Moreover, radius, ulna, and short bones are formed by endochondral ossification, while carpal bones are formed by intramembranous ossification, which is less dependent on growth hormones (Mari, 2015). When using the GP atlas, the phalanges and metacarpals are more emphasized (Larson et al., 2018). Nevertheless, even when images with such large deviations were included in the testing set, the overall accuracy of our Al system was nearly $85 \%$.

It has remained controversial whether Al would replace radiologists. Similar to the good performance of Al in detecting skin cancer (Esteva et al., 2017), diabetic retinopathy (Gulshan et al., 2016), and breast cancer (Rodríguez-Ruiz et al., 2019), various Al systems (Larson et al., 2018; Bui, Lee \& Shin, 2019; Liu et al., 2019) were reported to achieve comparable BA results to those of experienced readers. With the advantage of artificial neural networks and deep learning, Al can "see" (abstract) subtle imaging characteristics to learn and improve the algorithm automatically, thus overcoming the limitations of time, energy, expenses, and intraand inter-observer variations of human radiologists. However, Al algorithms for image 
284

285

286

287

288

289

290

291

292

293

294

295

296

297

recognition must be developed on the basis of "labeled data" where BA results interpreted by human radiologists are taken as the ground truth (Davenport \& Dreyer, 2018). Therefore, researchers are now heading toward the model in which both Al and radiologists can work cooperatively rather than competitively. It has been proven that readers with the assistance of Al can achieve better BA accuracy than readers alone or Al alone (Kim et al., 2017; Tajmir et al., 2019). In our study, radiologists could identify deformities and malposition from hand radiographs concurrently while giving the BA results, but these are technical obstacles for Al processing. In the future, a more precise BA workflow is expected that integrates Al with radiologists' practice.

Our research still had some limitations. First, the test sample size was still relatively small for an Al validation, but our number of patients was much larger than that of most prior studies and the largest one among Chinese population. Second, we did not investigate whether the Al system could help human reviewers to improve the accuracy of BA assessment, as the Al system was more likely to become an auxiliary method for human reviewers. Third, we did not test the performance of our algorithm on the RSNA dataset because our algorithm was developed using a different dataset with quite different imaging quality and demographic information. Lastly, more efforts should be made to improve the accuracy of this AI BA system and to broaden its age range.

\section{CONCLUSION}

The developed automated Al system could achieve comparable BA results to experienced reviewers for Chinese children with abnormal growth and development.

\section{Acknowledgment}

The authors thank Bo Liu (Hangzhou YITU Healthcare Technology Co., Ltd.) for his technical support and Dr. Huadan Xue (Department of Radiology, Peking Union Medical College Hospital, Chinese Academy of Medical Sciences \& Peking Union Medical College) and Dr. Daming Zhang (Department of Radiology, Peking Union Medical College Hospital, Chinese Academy of Medical 
312

313

314

315

316

317

318

319

320

321

322

323

324

325

326

327

328

329

330

331

332

333

334

335

336

337

338

Sciences \& Peking Union Medical College) for their help during proposal design.

\section{REFERENCES}

Alshamrani K, Messina F, Offiah AC. 2019. Is the Greulich and Pyle atlas applicable to all ethnicities? A systematic review and meta-analysis. Eurpean Radiology 29(6):2910-2923 DOI 10.1007/s00330-018-5792-5.

Booz C, Wichmann JL, Boettger S, Al KA, Martin SS, Lenga L, Leithner D, Albrecht MH, Ackermann H, Vogl T J, Bodelle B, Kaltenbach B. 2019. Evaluation of a Computer-Aided Diagnosis System for Automated Bone Age Assessment in Comparison to the Greulich-Pyle Atlas Method. Journal of Computer Assisted Tomography 43(1):39-45 DOI 10.1097/RCT.0000000000000786.

Bui TD, Lee JJ, Shin J. 2019. Incorporated region detection and classification using deep convolutional networks for bone age assessment. Artificial Intelligence in Medicine 97:1-8 DOI 10.1016/j.artmed.2019.04.005.

Bunch PM, Altes TA, Mcllhenny J, Patrie J, Gaskin CM. 2017. Skeletal development of the hand and wrist: digital bone age companion-a suitable alternative to the Greulich and Pyle atlas for bone age assessment? Skeletal Radiology 46(6):785-793 DOI 10.1007/s00256-017-26167.

Cheng CT, Ho TY, Lee TY, Chang CC, Chou CC, Chen CC, Chung IF, Liao CH. 2019. Application of a deep learning algorithm for detection and visualization of hip fractures on plain pelvic radiographs. European Radiology 29(10):5469-5477 DOI 10.1007/s00330-019-06167-y.

Creo AL, Schwenk WF. 2017. Bone age: a handy tool for pediatric providers. Pediatrics 140(6):e20171486 DOI 10.1542/peds.2017-1486.

Davenport T, Dreyer K. 2018. Al will change radiology, but it won't replace radiologists. Available via https://hbr.org/2018/03/ai-will-change-radiology-but-it-wont-replaceradiologists (accessed 20 August 2019).

De Sanctis V, Di Maio S, Soliman A, Raiola G, Elalaily R, Millimaggi G. 2014. Hand X-ray in 
339

340

pediatric endocrinology: skeletal age assessment and beyond. Indian Journal of Endocrinology and Metabolism 18(7):63-71 DOI 10.4103/2230-8210.145076.

Ebrahimzade M, Rahban D, Baboli Bahmaei A, Masoumi S, Changizi V, Mirdoraghi M. 2019. Evaluation of skeletal age based on Greulich-Pyle method in Tehran. MethodsX 6:1543-1546 DOI 10.1016/j.mex.2019.06.004.

Esteva A, Kuprel B, Novoa RA, Ko J, Swetter SM, Blau HM, Thrun S. 2017. Corrigendum: dermatologist-level classification of skin cancer with deep neural networks. Nature 546(7660):686 DOI 10.1038/nature22985.

Greulich WW, Pyle SI. 1959. Radiographic atlas of skeletal development of the hand and wrist, 2nd ed. Stanford, CA: Stanford University Press.

Gulshan V, Peng L, Coram M, Stumpe MC, Wu D, Narayanaswamy A, Venugopalan S, Widner K, Madams T, Cuadros J, Kim R, Raman R, Nelson PC, Mega JL, Webster DR. 2016. Development and validation of a deep learning algorithm for detection of diabetic retinopathy in retinal fundus photographs. JAMA 316(22):2402-2410 DOI 10.1001/jama.2016.17216.

Halabi SS, Prevedello LM, Kalpathy-Cramer J, Mamonov AB, Bilbily A, Cicero M, Pan I, Pereira LA, Sousa RT, Abdala N, Kitamura FC, Thodberg HH, Chen L, Shih G, Andriole K, Kohli MD, Erickson BJ, Flanders AE. 2019. The RSNA Pediatric Bone Age Machine Learning Challenge. Radiology 290(2):498-503 DOI 10.1148/radiol.2018180736.

Hosny A, Parmar C, Quackenbush J, Schwartz LH, Aerts HJWL. 2018. Artificial intelligence in radiology. Nature Reviews Cancer 18(8):500-510 DOI 10.1038/s41568-018-0016-5.

Hu TH, Wan L, Liu TA, Wang MW, Chen T, Wang YH. 2017. Advantages and application prospects of deep learning in image recognition and BA assessment. Fa Yi Xue Za Zhi 33(6):629-634 DOI 10.3969/j.issn.1004-5619.2017.06.013.

Hsieh CW, Jong TL, Chou YH, Tiu CM. 2007. Computerized geometric features of carpal bone for bone age estimation. Chinese Medcial Journal (English) 120(9):767-770

Kim HJ, Song HR, Shyam A, Heon SS, Unnikrishnan R, Song SY. 2010. Skeletal age in idiopathic 
366

367

368

369

370

371

372

373

374

375

376

377

378

379

380

381

382

383

short stature: an analytical study by the TW3 method, Greulich and Pyle method. Indian Journal of Orthopaedics 44(3):322-326 DOI 10.4103/0019-5413.65144.

Kim JR, Shim WH, Yoon HM, Hong SH, Lee JS, Cho YA, Kim S. 2017. Computerized Bone Age Estimation Using Deep Learning-Based Program: evaluation of the Accuracy and Efficiency. American Journal of Roentgenology 209(6):1374-1380 DOI 10.1001/jama.2016.17216.

Larson DB, Chen MC, Lungren MP, Halabi SS, Stence NV, Langlotz CP. 2018. Performance of a deep-learning neural network model in assessing skeletal maturity on pediatric hand radiographs. Radiology 287(1):313-322 DOI 10.1148/radiol.2017170236.

Liu J, Qi J, Liu Z, Ning Q, Luo X. 2008. Automatic bone age assessment based on intelligent algorithms and comparison with TW3 method. Computerized Medical Imaging and Graphics 32(8):678-684 DOI 10.1016/j.compmedimag.2008.08.005.

Liu Y, Zhang C, Cheng J, Chen X, Wang Z. 2019. A multi-scale data fusion framework for bone age assessment with convolutional neural networks. Computers in Biology and Medicine 108:161-173 DOI 10.1016/j.combiomed.2019.03.015.

Mari S. 2015. Bone age: assessment methods and clinical applications. Clinical Pediatric Endocrinology 24(4):143-152 DOI 10.1297/cpe.24.143

Molinari L, Gasser T, Largo RH. 2004. TW3 bone age: RUS/CB and gender differences of percentiles for score and score increments. Annals of Human Biology 31(4):421-435 DOI 10.1080/03014460410001723969.

Polito C, Greco N, Opallo A, Cimmaruta E, La Manna A. 1994. Alternate-day steroids affect carpal maturation more than radius, ulna and short bones. Pediatric Nephrology 8(4):480482 DOI 10.1007/bf00856538.

Ren X, Li T, Yang X, Wang S, Ahmad S, Xiang L, Stone SR, Li L, Zhan Y, Shen D, Wang Q. 2019. Regression convolutional neural network for automated pediatric bone age assessment from hand radiograph. IEEE Journal of Biomedical Health Informatics 23(5):2030-2038 DOI 10.1109/JBHI.2018.2876916.

Rodríguez-Ruiz A, Krupinski E, Mordang JJ, Schilling K, Heywang-Köbrunner SH, Sechopoulos I, 
393

394

395

396

397

398

399

400

401

402

403

404

405

406

407

408

409

410

411

412

413

414

415

416

417

418

Mann RM. 2019. Detection of breast cancer with mammography: effect of an artificial intelligence support system. Radiology 290(2):305-314 DOI 10.1148/radiol.201818371.

Siegel EL. 2019. What can we learn from the RSNA Pediatric Bone Age Machine Learning Challenge? Radiology 290(2):504-505 DOI 10.1148/radiol.2018182657.

Spampinato C, Palazzo S, Giordano D, Aldinucci M, Leonardi R. 2017. Deep learning for automated skeletal bone age assessment in X-ray images. Medical Image Analysis 36:41-51 DOI 10.1016/j.media.2016.10.010.

Tajmir SH, Lee H, Shailam R, Gale HI, Nguyen JC, Westra SJ, Lim R, Yune S, Gee MS, Do S. 2019. Artificial intelligence-assisted interpretation of bone age radiographs improves accuracy and decreases variability. Skeletal Radiology 48(2):275-283 DOI 10.1007/s00256-018-3033-2.

Tanner JM. 1962. Growth at adolescence: with a general consideration of the effects of hereditary and environmental factors upon growth and maturation from birth to maturity, 2nd ed. Springfield, IL: Blackwell Scientific Publications.

Tanner JM, Whitehouse RH, Cameron N, Marshall WA, Healy MJR, Goldstein H. 1983. Assessment of skeletal maturity and prediction of adult height (TW2 method), 2nd ed. Cambridge, MA: Academic Press.

Tanner JM, Healy MJR, Goldstein H, Cameron N. 2001. Assessment of skeletal maturity and prediction of adult height (TW3 method), 3rd ed. London, United Kingdom: WB Saunders.

Thodberg HH, van Rijn RR, Jenni OG, Martin DD. 2017. Automated determination of bone age from hand X-rays at the end of puberty and its applicability for age estimation. International Journal of Legal Medicine 131(3):771-780 DOI 10.1007/s00414-016-1471-8.

van Rijn RR, Thodberg HH. 2013. Bone age assessment: automated techniques coming of age? Acta Radiologica 54(9):1024-1029 DOI 10.1258/ar.2012.120443.

Zhang SY, Liu LJ, Han YS, Liu G, Ma ZG, Shen XZ, Xu RL, Hua JQ. 2008. Reference values of differences between TW3-C RUS and TW3-C carpal bone ages of children from five cities of China. Zhonghua Er Ke Za Zhi 46(11):851-855. 


\section{Table 1 (on next page)}

The demographic characteristics of the 745 Chinese patients 
1 Table 1 The demographic characteristics of the 745 Chinese patients

2

\begin{tabular}{|c|c|c|c|}
\hline \multicolumn{2}{|c|}{ Characteristics } & \multicolumn{2}{|l|}{ Number (\%) } \\
\hline \multirow[t]{2}{*}{ Gender } & Male & $360(48.32 \%)$ & \\
\hline & Female & $385(51.58 \%)$ & \\
\hline Age & 4 & $21(2.82 \%)$ & \\
\hline \multirow[t]{14}{*}{ (years) } & 5 & $59(7.92 \%)$ & $126(16.91 \%)$ \\
\hline & 6 & $46(6.16 \%)$ & \\
\hline & 7 & $53(7.11 \%)$ & \multirow{6}{*}{$420(56.38 \%)$} \\
\hline & 8 & $46(6.17 \%)$ & \\
\hline & 9 & $118(15.84 \%)$ & \\
\hline & 10 & $68(9.13 \%)$ & \\
\hline & 11 & $70(9.40 \%)$ & \\
\hline & 12 & $65(8.72 \%)$ & \\
\hline & 13 & $43(5.77 \%)$ & \multirow{6}{*}{$199(26.71 \%)$} \\
\hline & 14 & $78(10.47)$ & \\
\hline & 15 & $32(4.30 \%)$ & \\
\hline & 16 & $26(3.49 \%)$ & \\
\hline & 17 & $13(1.74 \%)$ & \\
\hline & 18 & $7(0.94 \%)$ & \\
\hline \multirow[t]{20}{*}{ Diagnosis } & Growth hormone deficiency & $214(28.72 \%)$ & \\
\hline & Turner syndrome & $81(10.87 \%)$ & \\
\hline & Precocious puberty & $77(10.34 \%)$ & \\
\hline & Congenital adrenal hyperplasia & $72(9.66 \%)$ & \\
\hline & Hypogonadism & $25(3.36 \%)$ & \\
\hline & Small for gestational age & $22(2.95 \%)$ & \\
\hline & Kallmann's syndrome & $15(2.01 \%)$ & \\
\hline & Hypothyroidism & $13(1.74 \%)$ & \\
\hline & Hypospadias & $10(1.34 \%)$ & \\
\hline & Mixed gonadal dysgenesis & $9(1.21 \%)$ & \\
\hline & Noonan syndrome & $6(0.81 \%)$ & \\
\hline & Prader-willi syndrome & $5(0.67 \%)$ & \\
\hline & Cryptorchidism & $5(0.67 \%)$ & \\
\hline & Systemic juvenile idiopathic arthritis & $5(0.67 \%)$ & \\
\hline & Renal tubular acidosis & $5(0.67 \%)$ & \\
\hline & McCune-Albright syndrome & $3(0.40 \%)$ & \\
\hline & Pseudohypoparathyroidism & $2(0.27 \%)$ & \\
\hline & Gynecomastia & $2(0.27 \%)$ & \\
\hline & Gigantism & $1(0.13 \%)$ & \\
\hline & Short stature for unknown reasons & $173(23.22 \%)$ & \\
\hline
\end{tabular}




\section{Table 2 (on next page)}

Clinical features of the five cases with the largest BA deviation ( $\geq 2$ years) between $\mathrm{Al}$ and the reference standard 
1 Table 2 Clinical features of the five cases with the largest BA deviation ( $\geq 2$ years) between

$2 \quad \mathrm{Al}$ and the reference standard

\begin{tabular}{ccclcc}
\hline $\begin{array}{c}\text { Patient } \\
\text { Number }\end{array}$ & Sex & $\begin{array}{c}\text { Chronological } \\
\text { age (years) }\end{array}$ & \multicolumn{1}{c}{ Diagnosis } & \multicolumn{2}{c}{ Bone age (years) } \\
\cline { 3 - 5 } 1 & F & 8.2 & Idiopathic short stature & 8.8 & 11.1 \\
2 & M & 13.4 & Growth hormone deficiency & 15 & 12.5 \\
3 & F & 13.4 & Systemic juvenile idiopathic arthritis & 9 & 11.7 \\
4 & M & 10.5 & Idiopathic short stature & 9 & 7 \\
5 & M & 7.3 & Turner syndrome & 4.5 & 2.1 \\
\hline
\end{tabular}

3 


\section{Figure 1}

Network structure of the region-based convolutional neural network.

The left hand radiograph is preprocessed, and the alignment module then adopts a deep residual network (ResNet) to localize the bounding area of the targeted bones. Subsequently, the classification module also adopts a ResNet for extracting features of targeted bones and provides bone age result.

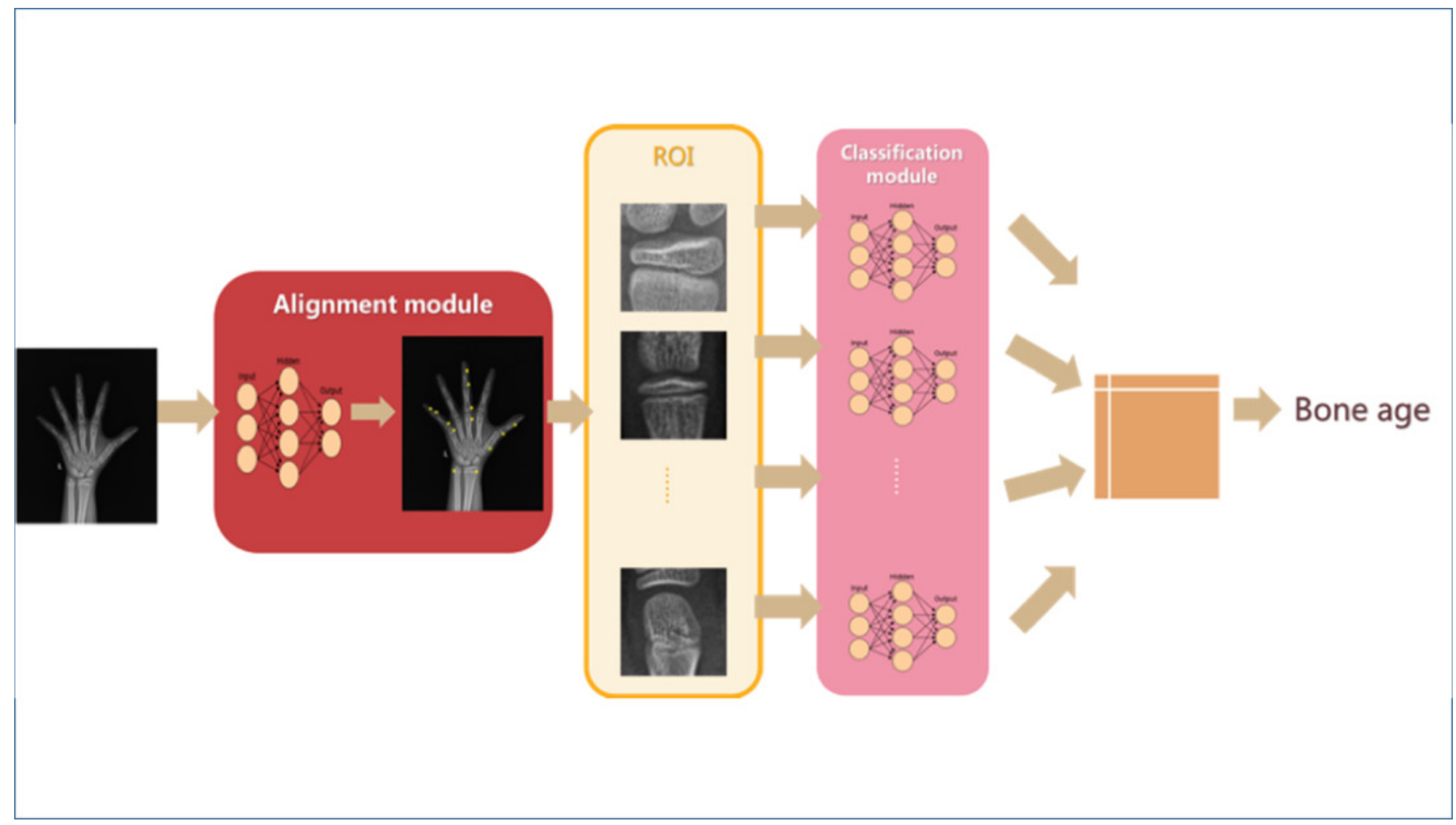


Figure 2

The flowchart of cohort selection with inclusion and exclusion criteria. 


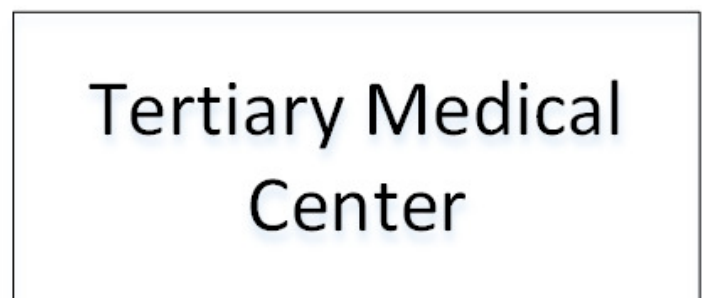

Bone age radiographs matching the following conditions:

1. left hand images

2. January and October 2018

3. Age between 4 and 18 years

\section{Cases}

8 Cases Excluded for
Poor Image Quality

\section{Cases: 360 boys 385 girls}


Figure 3

The accuracy of Al BA determined by comparing with the reference standard for different age groups.

Distribution of BA accuracy within 1 year is shown according to chronological age $(A)$ and three age groups (4-6, 6-12, and 12-18 years) (B).

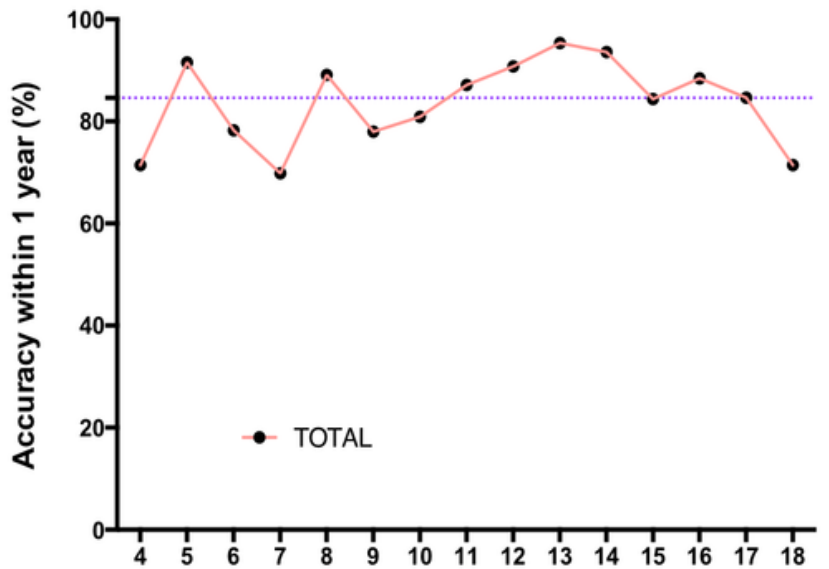

A

Age groups

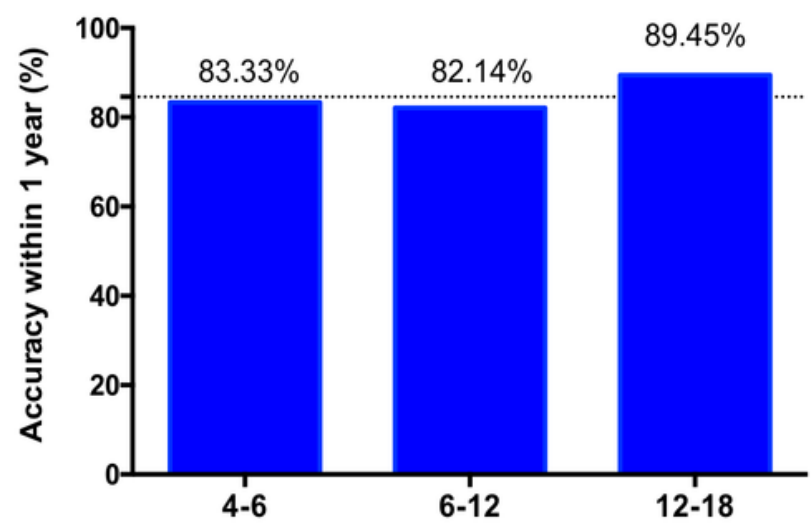

B

Age groups (years) 


\section{Figure 4}

Examples of left hand bone age (BA) radiographs from four different patients.

A. Left hand radiograph from a 9.3-year-old girl with precocious puberty. Both the manual and $\mathrm{Al} \mathrm{BA}$ result is 10 years. B. Left hand radiograph from an 8.2-year-old girl with idiopathic short stature. The manual BA result is 8.8 years, and the AI BA result is 11.1 years. The fifth middle phalange is short and without a normal epiphysis. C. Left hand radiograph from a 13.4-year-old girl with systemic juvenile idiopathic arthritis and receiving long-term treatment with corticosteroids. The manual BA result is 9 years, and the AI BA result is 11.7 years. There is severe osteomalacia of hand and wrist bones, and the shapes of the carpal bones are irregular. D. Left hand radiograph from a 7.3-year-old boy with Turner syndrome. The manual BA result is 4.5 years, and the AI BA result is 2.1 years. The different rates of maturation of the carpals and phalanges may cause this deviation in the results. 


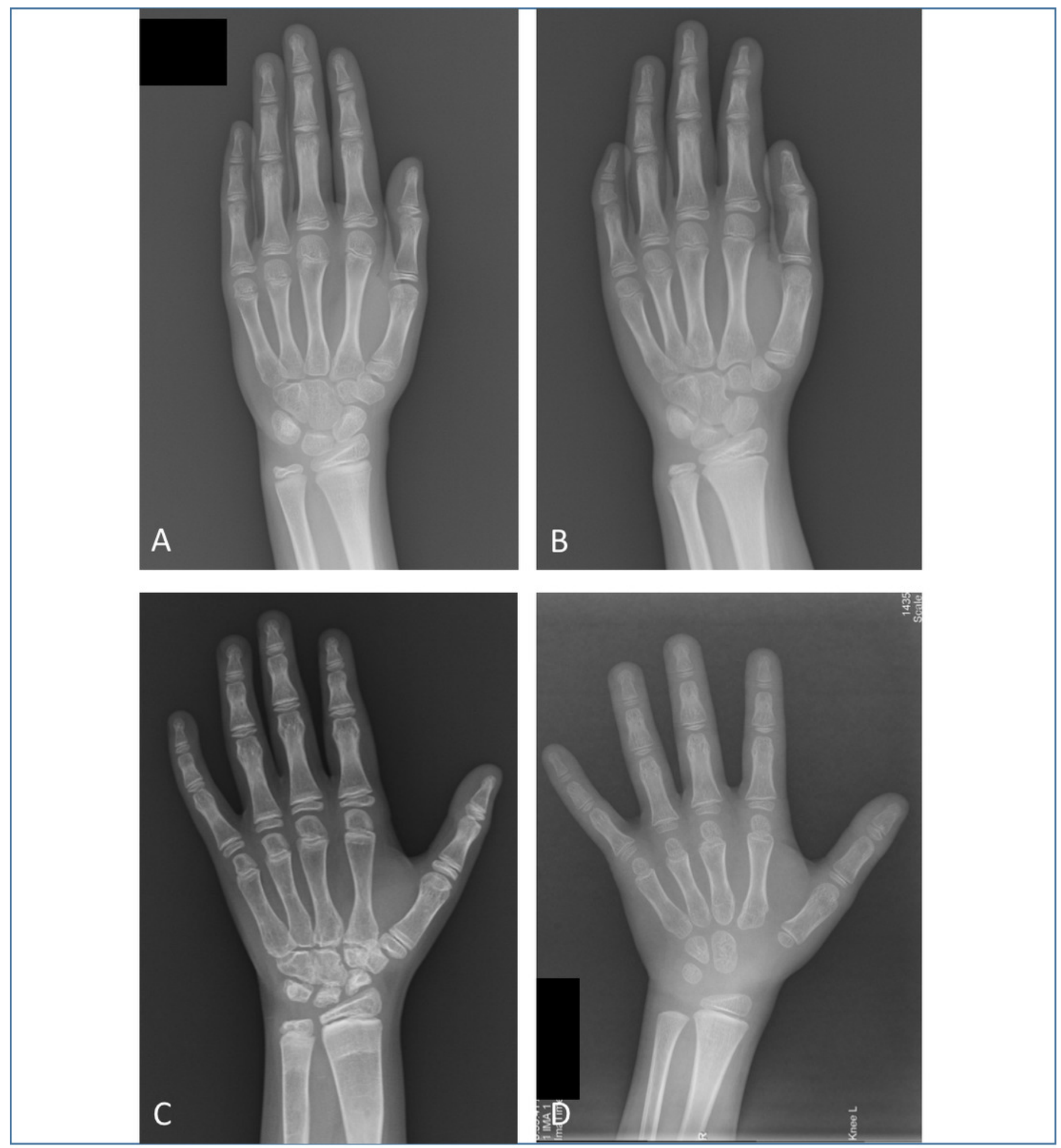


Figure 5

The Bland-Altman plot showing the difference between the AI BA and the reference standard.

\section{Bland-Altman}

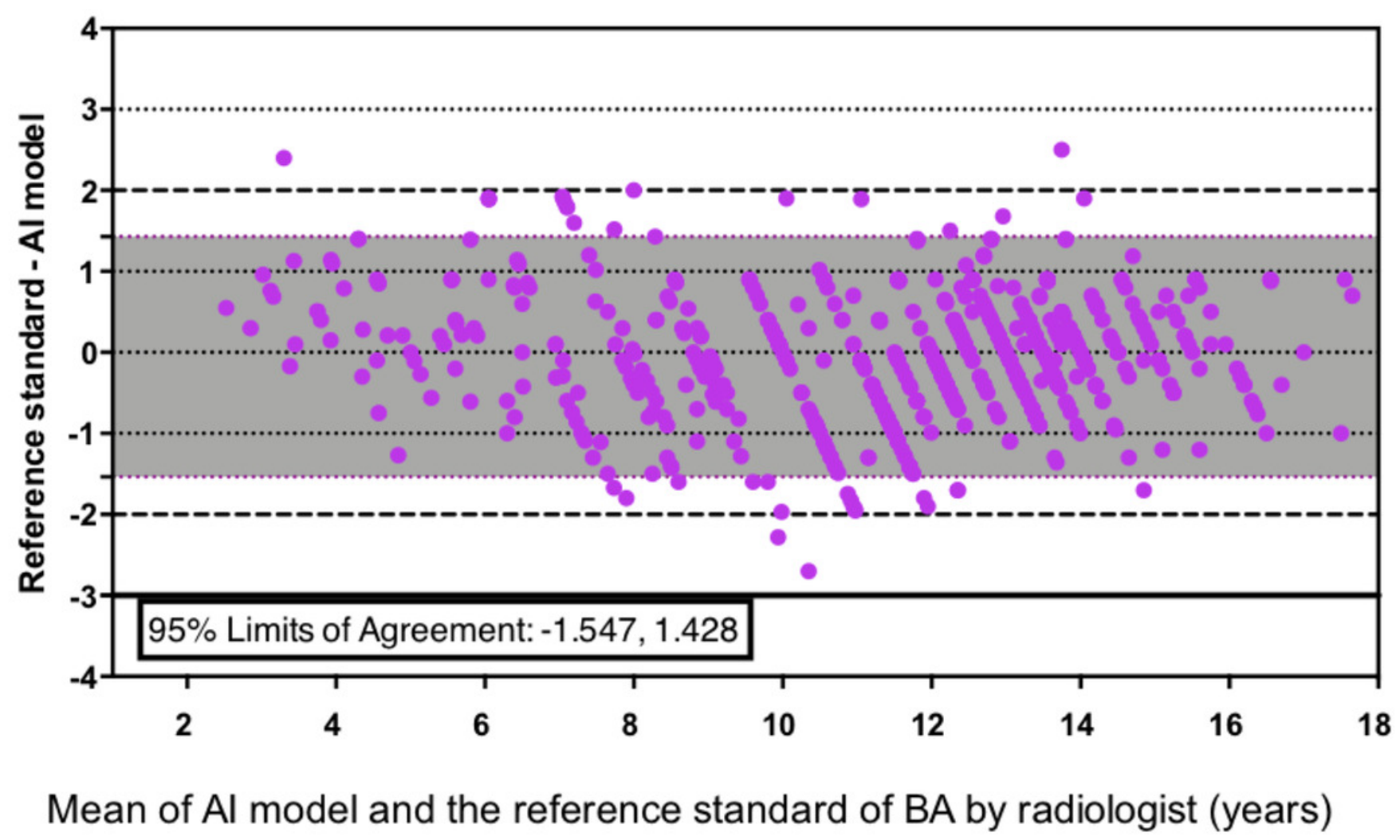

